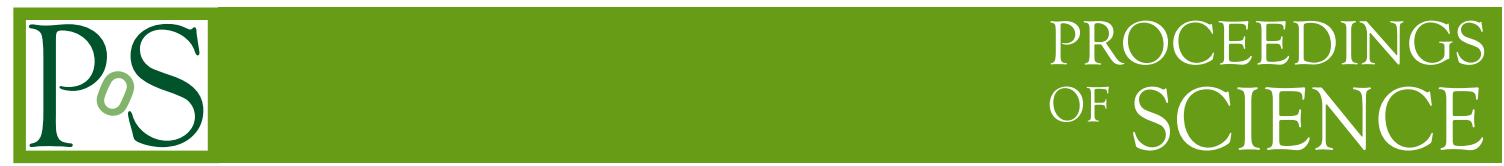

\title{
Conventional versus unconventional nature of X(3915)
}

\section{Pedro Gonzalez*}

Universitat de Valencia and IFIC (CSIC)

E-mail: pedro.gonzalez@uv.es

We compare the results for electromagnetic and strong decays of $X(3915)$ from two nonrelativistic quark model descriptions: a conventional one in terms of the Cornell potential and an unconventional one from a Generalized Screened potential. We conclude that the experimental significance of the OZI forbidden decay $X(3915) \rightarrow \omega J / \psi$ favors an unconventional description.

VIII International Workshop On Charm Physics

5-9 September, 2016

Bologna, Italy

${ }^{*}$ Speaker. 


\section{Introduction}

In the 2012 edition of the Review of Particle Physics [1] a $0^{+}\left(?^{?+}\right)$ unconventional charmonium state named $X(3915)$ was listed. Two years later, in the 2014 edition [2], a $0^{+}\left(0^{++}\right)$ conventional charmonium state $\chi_{c 0}(2 p)$ substituted it (conventional means that it can be described as a quark-antiquark state from a Cornell potential plus corrections [3, 4]). This new assignment has been a matter of debate: in references $[5,6]$ it has been argued that the mass, width, decay properties and production rates are incompatible with a $\chi_{c 0}(2 p)$ state. In fact, alternative descriptions of the $X$ (3915) -meson-antimeson molecule, tetraquark, mixed charmonium-molecule...-, have been developed (see [7] and references therein) yet not fully compatible with data.

In this talk a comparative study of the $X(3915) \rightarrow D \bar{D}$ and $X(3915) \rightarrow \omega J / \psi$ strong decays and the $X(3915) \rightarrow \gamma \gamma$ electromagnetic decay, from a conventional Cornell model and an unconventional Generalized Screened Potential Model [8, 9], will be presented. The comparison of the results obtained to data will be used to discriminate between both model descriptions of the $X(3915)$.

\section{Quark model descriptions}

The Cornell model is based on the quark-antiquark potential [3]

$$
V_{C o r}(r) \equiv \sigma r-\frac{\zeta}{r} \quad(r: 0 \rightarrow \infty)
$$

with $r$ standing for the quark-antiquark distance and the parameters $\sigma$ and $\zeta$ for the string tension and the color coulomb strength respectively. This model and refined versions of it [4] have been very successful in the description of the heavy quarkonia spectra below the open-flavor two meson thresholds. Above these thresholds the effect of two open flavor meson channels have been explicitly implemented $[10,11]$ but a good overall description of data seems difficult to be attained.

In the Generalized Screened Potential Model (GSPM) [8, 9], also based on a quark-antiquark structure, an effective quark-antiquark static potential $V(r)$ that implicitly incorporates threshold effects, in particular color screening from open flavor meson-meson configurations in the way suggested by lattice QCD [12], is considered. The model has been applied to heavy quarkonia showing that a reasonable overall spectral description of $J^{++}$resonances below and above thresholds (to which the model can be applied at its present stage) can be achieved.

By calling $M_{T_{i}}$ with $i \geq 1$ the masses of the physical open flavor meson-meson thresholds, $T_{i}$, with a given set of quantum numbers $I\left(J^{P C}\right)$, and defining $M_{T_{0}} \equiv 0$ for a unified notation (note that $T_{0}$ does not correspond to any physical meson-meson threshold), the form of $V(r)$ in the different energy regions (specified as energy interval subindices) reads:

$$
V_{\left[M_{T_{0}}, M_{T_{1}}\right]}(r)= \begin{cases}\sigma r-\frac{\zeta}{r} & r \leq r_{T_{1}} \\ M_{T_{1}}-m_{Q}-m_{\bar{Q}} & r \geq r_{T_{1}}\end{cases}
$$




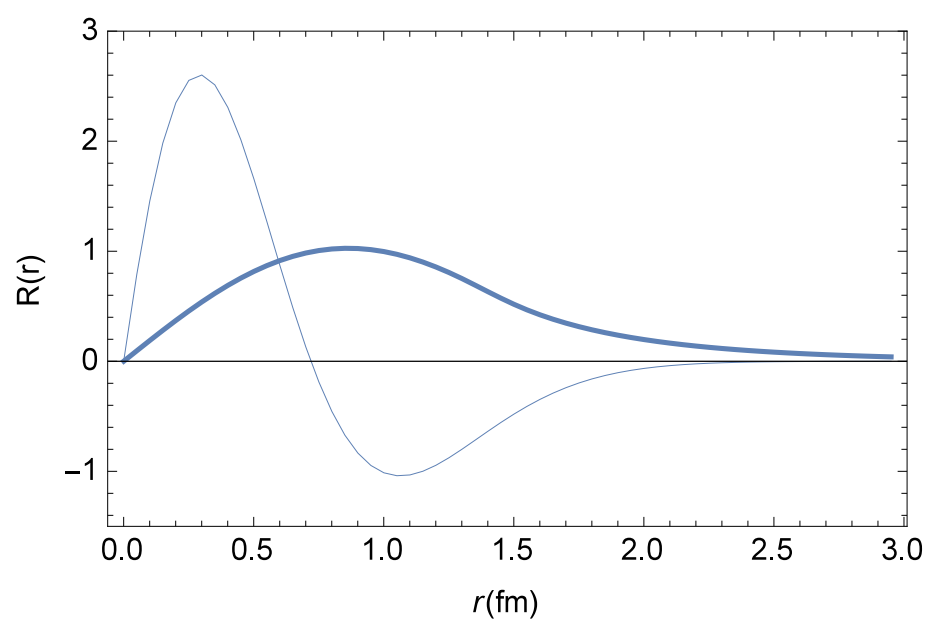

Figure 1: Radial wave functions R(r) (in units $\mathrm{fm}^{-\frac{3}{2}}$ ) for the $0^{++}\left(1 p_{\left[T_{1}, T_{2}\right]}\right)$ GSPM state (thick line) and the $0^{++}(2 p)$ Cornell state (thin line).

and

$$
V_{\left[M_{\left.T_{j-1}, M_{T_{j}}\right]}\right.}(r)=\left\{\begin{array}{lr}
M_{T_{j-1}}-m_{Q}-m_{\bar{Q}} & r \leq r_{T_{j-1}} \\
\sigma r-\frac{\zeta}{r} & r_{T_{j-1}} \leq r \leq r_{T_{j}} \\
M_{T_{j}}-m_{Q}-m_{\bar{Q}} & r \geq r_{T_{j}}
\end{array}\right.
$$

for $j>1$, where $m_{Q}\left(m_{\bar{Q}}\right)$ stands for the mass of the heavy quark (antiquark) and with the crossing radii $r_{T_{i}}(i \geq 1)$ defined by the continuity of the potential as

$$
\sigma r_{T_{i}}-\frac{\zeta}{r_{T_{i}}}=M_{T_{i}}-m_{Q}-m_{\bar{Q}}
$$

Thus, $V(r)$ has in each energy region between neighbor thresholds a Cornell form but modulated at short and long distances by these thresholds.

From (2.2) it is clear that the description of states far below the lowest threshold $M_{T_{1}}$ is going to be identical to the Cornell one; however, a completely different description of the states above $M_{T_{1}}$ comes out. For instance, the $0^{+}\left(0^{++}\right)$bound state in the energy region $\left[M_{T_{1}=D^{0} \bar{D}^{0}}, M_{T_{2}=D_{s}^{+} D_{s}^{-}}\right]$ is obtained by solving the Schrödinger equation for $V_{\left[M_{D^{0} \bar{D}^{0}}, M_{D_{s}^{+} D_{s}^{-}}\right]}(r)$. This $1 p_{\left[T_{1}, T_{2}\right]}$ GSPM state with calculated mass $3897.9 \mathrm{MeV}$ which should be assigned to $X(3915)$, differs greatly from the $2 p$ Cornell one as can be checked in Fig. 1 from reference [9] where the respective radial wave functions are plotted.

3. $X(3915) \rightarrow D \bar{D}$

Existing data indicate that this decay it is suppressed in spite of the fact that it is OZI allowed. In the quark model framework two different approaches have been developed to deal with this decay: the ${ }^{3} P_{0}$ decay model [13] where a $q \bar{q}$ is created in the hadronic vacuum with $0^{++}$quantum 


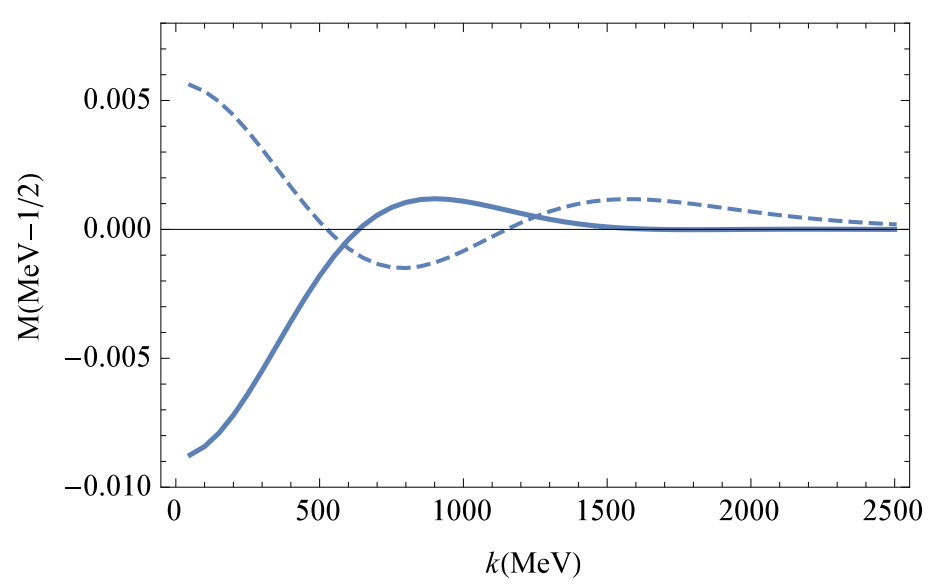

Figure 2: Momentum dependence of the ${ }^{3} P_{0}$ decay ampitude for the GSPM (solid line) and Cornell (dashed line) descriptions of $X(3915)$.

numbers and the $C^{3}$ (Cornell Coupled-Channel) decay model [3] where the $q \bar{q}$ creation is governed by the same potential generating the spectrum. Both models give a reasonable description of $I\left(J^{P C}\right)$ charmonium decays below the corresponding first open flavor meson-meson thresholds. Detailed expressions for the decay amplitude $A$ of a $c \bar{c}$ meson to $D \bar{D}$ in both models have been given elsewhere $[3,14]$. Although the momentum of $D($ and $\bar{D})$ is fixed $\left(k_{D}=599.6 \mathrm{MeV}\right)$ it is convenient to keep it as a variable and examine the amplitude dependence on it. Thus, by assuming that non considered momentum dependent corrections (relativistic terms, vertex momentum dependences...) may be effectively parametrized as a shift in the momentum, one may have an idea of the possible importance of such corrections.

In Figs. 2 and 3 the results obtained for $M \equiv \frac{A_{3_{P_{0}}}}{\gamma}$ where the constant $\gamma$ specifies the strength of the pair creation and $G \equiv A_{C^{3}}$ are plotted.For $X$ (3915) the wave functions from the Cornell and GSPM descriptions are used; for $D$ and $\bar{D}$ the usual gaussian approximation wave function (see for example [3]) is taken.

As can be checked from Fig. 2, for the GSPM description the ${ }^{3} P_{0}$ amplitude vanishes for a value of $k=637 \mathrm{MeV}$ close to $k_{D}=599.6 \mathrm{MeV}$. Hence it is plausible that momentum dependent corrections to the ${ }^{3} P_{0}$ decay model make the amplitude to vanish. On the other hand, as can be checked from Fig. 3, for the Cornell description the $C^{3}$ amplitude vanishes for a value of $k=558 \mathrm{MeV}$ close to $k_{D}=599.6 \mathrm{MeV}$. Hence it is plausible that momentum dependent corrections to the $C^{3}$ decay model make the amplitude to vanish. We may then conclude that the observed suppression of the decay $X(3915) \rightarrow D \bar{D}$ might be equally well explained from a $C^{3}$ decay model with a Cornell description of $X(3915)$ and from a ${ }^{3} P_{0}$ decay model with a GSPM description of $X(3915)$. Therefore, no conclusion about the conventional or unconventional nature of $X(3915)$ can be extracted from its decay to $D \bar{D}$.

\section{The $X(3915) \rightarrow \omega J / \psi$ decay}

Experimental information on this decay comes from the average of measured production rates 


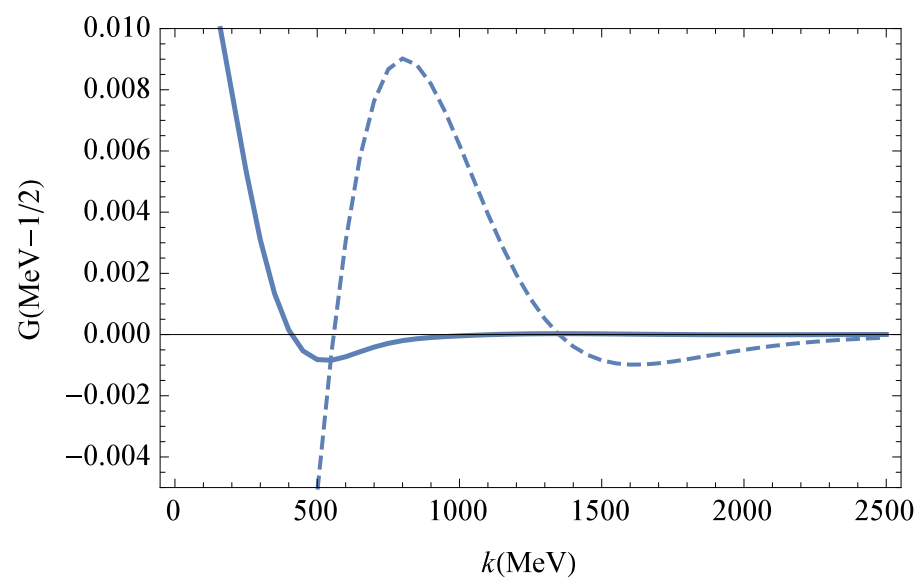

Figure 3: Momentum dependence of the $C^{3}$ decay ampitude for the GSPM (solid line) and Cornell (dashed line) descriptions of $X(3915)$.

in two-photon fusion [2]

$$
\Gamma(X(3915) \rightarrow \gamma \gamma) \mathscr{B}(X(3915) \rightarrow J / \psi \omega)=54 \pm 9 \mathrm{eV}
$$

and from the average of the product of branching fraction measurements for $X(3915)$ production in $B$ decay (see [6] and references therein)

$$
\mathscr{B}\left(B^{+} \rightarrow K^{+} X(3915)\right) \mathscr{B}(X(3915) \rightarrow J / \psi \omega)=3.0_{-0.5-0.3}^{+0.6+0.5} \times 10^{-5}
$$

In reference [6] it has been argued that if $X(3915)$ were a $\chi_{c 0}(2 p)$ Cornell state then it would be reasonable to assume $\mathscr{B}\left(B^{+} \rightarrow K^{+} \chi_{c 0}(2 p)\right) \lesssim \mathscr{B}\left(B^{+} \rightarrow K^{+} \chi_{c 0}(1 p)\right)$. Then, using the experimental value $\mathscr{B}\left(B^{+} \rightarrow K^{+} \chi_{c 0}(1 p)\right)=1.5_{-0.14}^{+0.15} \times 10^{-4}$ one would get from (4.2)

$$
\mathscr{B}\left(\chi_{c 0}(2 p) \rightarrow J / \psi \omega\right)>0.14
$$

On the other hand $\Gamma(X(3915) \rightarrow \gamma \gamma)$ is known to be proportional to square of the derivative of the radial wave function at the origin $\left|R_{X(3915)}^{\prime}(0)\right|^{2}[15]$. Therefore, if $X(3915)$ were a $\chi_{c 0}(2 p)$ Cornell state we would expect $\Gamma\left(\chi_{c 0}(2 p) \rightarrow \gamma \gamma\right) \simeq 1.4 \Gamma\left(\chi_{c 0}(1 p) \rightarrow \gamma \gamma\right)$. Then, using the experimental value $\Gamma\left(\chi_{c 0}(1 p) \rightarrow \gamma \gamma\right)=2.3 \pm 0.4 \mathrm{KeV}$ one would get $\Gamma\left(\chi_{c 0}(2 p) \rightarrow \gamma \gamma\right) \sim 3.3 \pm 0.6 \mathrm{KeV}$. However, the combination of this value with (4.1) would give

$$
\mathscr{B}\left(\chi_{c 0}(2 p) \rightarrow J / \psi \omega\right) \sim 0.017 \pm 0.006
$$

which is clearly incompatible with (4.3).

Let us now consider the GSPM description, say $X(3915)$ is a $1 p_{\left[T_{1}, T_{2}\right]}$ GSPM state. By using again the assumption $\mathscr{B}\left(B^{+} \rightarrow K^{+} \chi_{c 0}(2 p)\right) \lesssim \mathscr{B}\left(B^{+} \rightarrow K^{+} \chi_{c 0}(1 p)\right)$ an upper bound for $\mathscr{B}\left(B^{+} \rightarrow K^{+} X_{1 p_{\left[T_{1}, T_{2}\right]}}\right)$ can be found. Taking into account that the phase space is the same for the 
GSPM and the Cornell descriptions we get from [16]

$$
\begin{aligned}
\mathscr{B}\left(B^{+} \rightarrow K^{+} X_{1 p_{\left[T_{1}, T_{2}\right]}}\right) & =\left(\frac{a+e\left(H_{1}\right)_{1 p_{\left[T_{1}, T_{2}\right]}}}{a+e\left(H_{1}\right)_{c 0}(2 p)}\right) \mathscr{B}\left(B^{+} \rightarrow K^{+} \chi_{c 0}(2 p)\right) \\
& \lesssim\left(\frac{a+0.1 \mathrm{MeV}}{a+7.1 \mathrm{MeV}}\right) \mathscr{B}\left(B^{+} \rightarrow K^{+} \chi_{c 0}(1 p)\right)
\end{aligned}
$$

where $a$ is an unknown constant to be determined phenomenologically and the values for $H_{1}$ and $e$ have been obtained from their expressions in [16].

Let us consider now $\Gamma\left(1 p_{\left[T_{1}, T_{2}\right]} \rightarrow \gamma \gamma\right)$. By using the relation to the square of the derivative of the radial wave function at the origin we obtain $\Gamma\left(1 p_{\left[T_{1}, T_{2}\right]} \rightarrow \gamma \gamma\right) \simeq 0.02 \Gamma\left(\chi_{c 0}(1 P) \rightarrow \gamma \gamma\right)$.

Using the experimental value for $\Gamma\left(\chi_{c 0}(1 P) \rightarrow \gamma \gamma\right)$ one gets $\Gamma\left(0^{++}\left(1 p_{\left[T_{1}, T_{2}\right]}\right) \rightarrow \gamma \gamma\right) \simeq 46 \pm 8$ $\mathrm{eV}$. Therefore, if $X(3915)$ is a $1 p_{\left[T_{1}, T_{2}\right]}$ GSPM state, the combination of this value with (4.1) gives

$$
\mathscr{B}\left(1 p_{\left[T_{1}, T_{2}\right]} \rightarrow J / \psi \omega\right)>0.83
$$

This implies from (4.2) that $\mathscr{B}\left(B^{+} \rightarrow K^{+} X_{1 p_{\left[T_{1}, T_{2}\right]}}\right)<3.6_{-0.6-0.4}^{+0.7+0.6} \times 10^{-5}$. Hence making this bound equal to the one previously obtained (4.5), we get a phenomenological value for $a$ compatible with data. For the central experimental value $\mathscr{B}\left(B^{+} \rightarrow K^{+} X_{1 p_{\left[T_{1}, T_{2}\right]}}\right)<3.6 \times 10^{-5}$ we obtain $a \sim 2.1 \mathrm{MeV}$. Hence a full consistent description of data is feasible.

\section{Summary}

A comparative study of the strong decays $X(3915) \rightarrow D \bar{D}$ and $X(3915) \rightarrow J / \psi \omega$ has been carried out from two quark model descriptions of $X(3915)$ : a conventional one from the well known Cornell potential and an unconventional from a Generalized Screened Potential Model (GSPM).

The $X(3915) \rightarrow D \bar{D}$ process has been studied from two decay models, the ${ }^{3} P_{0}$ and the $C^{3}$ (Cornell Coupled-Channel), usually employed within the quark model framework. The results obtained imply that no discrimination between the two descriptions employed can be done from this decay once momentum dependent corrections are taken into account.

A different situation may occur for $X(3915) \rightarrow J / \psi \omega$. We have shown that an explanation of existing data involving the branching fraction $\mathscr{B}(X(3915) \rightarrow J / \psi \omega)$ seems to be impossible to attain from the Cornell description. On the contrary, the GSPM description might accommodate all the experimental information predicting a quite big branching ratio for this OZI non allowed decay. The experimental confirmation of this prediction would clearly point out to a non conventional nature of $X(3915)$ putting in question the $\chi_{c 0}(2 p)$ PDG assignment.

This work has been supported by Ministerio de Economía y Competitividad of Spain grant FPA2013-47443-C2-1-P, by SEV-2014-0398 and by PrometeoII/2014/066 from Generalitat Valenciana. 


\section{References}

[1] K. A. Olive et al. [Particle Data Group (PDG)], Chin. Phys. C 38, 090001 (2014).

[2] K. A. Olive et al. [Particle Data Group (PDG)], Chin. Phys. C 38, 090001 (2014).

[3] E. Eichten, K. Gottfried, T. Kinoshita, K. D. Lane and T. M. Yan, Phys. Rev. D 17, 3090 (1978); Phys. Rev. D 21, 203 (1980).

[4] S. Godfrey and N. Isgur, Phys. Rev. D 32, 189 (1985).

[5] F. K. Guo and U. G. Meissner, Phys. Rev. D 86, 091501 (2012).

[6] S. L. Olsen, Phys. Rev. D 91, 057501 (2015).

[7] H-X. Chen, W. Chen, X. Liu and S-L. Zhu, Phys. Rep. 639, 1 (2016).

[8] P. González, J.Phys. G 41, 095001 (2014).

[9] P. González, Phys. Rev. D 92, 014017 (2015).

[10] E. Eichten, K. Lane and C. Quigg, Phys. Rev. D 69, 094019 (2004).

[11] E. Eichten, K. Lane and C. Quigg, Phys. Rev. D 75, 014014 (2006).

[12] G. S. Bali, H. Neff, T. Düssel, T. Lippert and K. Schilling (SESAM Collaboration), Phys. Rev. D 71, 114513 (2005).

[13] A. Le Yaouanc, L. Oliver, O. Pène and J. C. Raynal in "Hadron transitions in the quark model", Gordon and Breach Science Publishers 1988; Phys. Rev. D 8, 2223 (1973).

[14] S. Ono, Phys. Rev. D 23, 1118 (1981).

[15] W. Kuong, P. B. Mackenzie, R. Rosenfeld and J. L. Rosner, Phys. Rev. D 37, 3210 (1988).

[16] G. T. Bodwin, E. Braaten, T. C. Yuan and G. P. Lepage, Phys. Rev. D 46, R3703 (1992). 